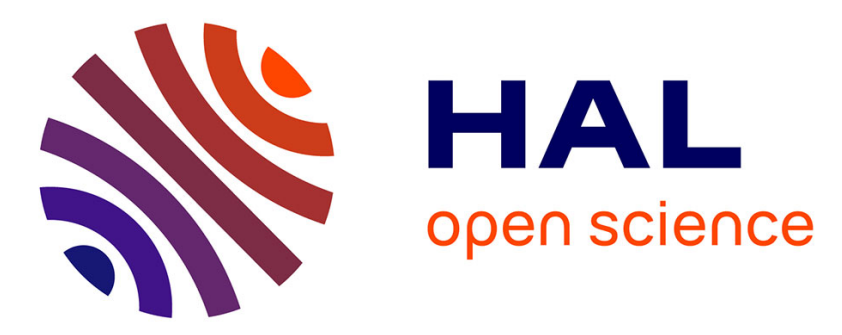

\title{
Analysis of Apparent Topography scheme for the linear wave equation with Coriolis force
}

\author{
Emmanuel Audusse, Minh-Hieu Do, Pascal Omnes, Yohan Penel
}

\section{To cite this version:}

Emmanuel Audusse, Minh-Hieu Do, Pascal Omnes, Yohan Penel. Analysis of Apparent Topography scheme for the linear wave equation with Coriolis force. FVCA 2017 - Finite Volumes for Complex Applications VIII, Jun 2017, Lille, France. pp.209-217, 10.1007/978-3-319-57394-6_23 . hal-01690796

\section{HAL Id: hal-01690796 \\ https://hal.science/hal-01690796}

Submitted on 23 Jan 2018

HAL is a multi-disciplinary open access archive for the deposit and dissemination of scientific research documents, whether they are published or not. The documents may come from teaching and research institutions in France or abroad, or from public or private research centers.
L'archive ouverte pluridisciplinaire $\mathbf{H A L}$, est destinée au dépôt et à la diffusion de documents scientifiques de niveau recherche, publiés ou non, émanant des établissements d'enseignement et de recherche français ou étrangers, des laboratoires publics ou privés. 


\title{
Analysis of Apparent Topography scheme for the linear wave equation with Coriolis force
}

Emmanuel Audusse, Minh Hieu Do, Pascal Omnes, Yohan Penel

\begin{abstract}
The shallow water equations can be used to model many phenomena in geophysical fluid mechanics. For large scales, the Coriolis force plays an important role and the geostrophic equilibrium which corresponds to the balance between the pressure gradient and the Coriolis force is an important feature. In this communication, we investigate the stability condition and the behavior of the so-called Apparent Topography scheme which is capable of capturing a discrete version of the geostrophic equilibrium.
\end{abstract}

Key words: Shallow water flows, Finite Volume Method, Coriolis Force, Wellbalanced Schemes.

MSC (2010): 65M08, 65M12, 76U05

Emmanuel Audusse, Minh Hieu Do, Pascal Omnes

Université Paris 13, Sorbonne Paris Cité, LAGA, CNRS UMR 7539,

99 av. J.-B. Clément, 94430 Villetaneuse, France

e-mail: audusse@math.univ-paris13.fr, do@math.univ-paris13.fr, omnes@math.univ-paris13.fr

Pascal Omnes

CEA Saclay, DEN, DM2S, STMF, LMSF

91191 Gif-sur-Yvette Cedex, France

e-mail: pascal.omnes@cea.fr

Yohan Penel

Team ANGE, Inria-Paris, CEREMA, UPMC and CNRS

2 rue Simone Iff, CS 42112, 75589 Paris Cedex 12, France

e-mail: yohan.penel@inria.fr 


\section{Introduction}

In order to study the Shallow Water equations with Coriolis source term, we consider the dimensionless formulation on the rotating frame which is given by

$$
\left\{\begin{array}{l}
\operatorname{St} \partial_{t} h+\nabla \cdot(h \overline{\mathbf{u}})=0, \\
\operatorname{St} \partial_{t}(h \overline{\mathbf{u}})+\nabla \cdot(h \overline{\mathbf{u}} \otimes \overline{\mathbf{u}})+\frac{1}{\mathrm{Fr}^{2}} \nabla\left(\frac{h^{2}}{2}\right)=-\frac{1}{\mathrm{Fr}^{2}} h \nabla b-\frac{1}{\mathrm{Ro}} h \overline{\mathbf{u}}^{\perp},
\end{array}\right.
$$

where unknowns $h$ and $\overline{\mathbf{u}}$ respectively denote the water depth and the average velocity over the water column and function $b(\mathbf{x})$ denotes the topography of the considered oceanic basin and is a given function. Dimensionless numbers St, Fr and Ro respectively stand for the Strouhal, the Froude and the Rossby numbers defined below. In the sequel, we shall focus on cases where

$$
\text { St }:=\frac{L}{U T}=\mathscr{O}\left(\frac{1}{\mathrm{M}}\right), \quad \text { Fr }:=\frac{U}{\sqrt{g H}}=\mathscr{O}(\mathrm{M}), \quad \text { Ro }:=\frac{U}{\Omega L}=\mathscr{O}(\mathrm{M}),
$$

with $\mathrm{M}$ a small parameter. The parameters $g$ and $\Omega$ denote the gravity coefficient and the angular velocity of the Earth. Constants $U, H, L$ and $T$ are some characteristic velocity, vertical and horizontal lengths and time. These orders of magnitude correspond to the study of short-time dynamics and standard conditions for large scale oceanic flows.

For data independent of $y$ and with a flat topography, the solution of System (1) then satisfies at the leading order the quasi-1d linear wave equation with Coriolis source term (see [2] for the derivation)

$$
\left\{\begin{array}{l}
\partial_{t} r+a_{\star} \partial_{x} u=0 \\
\partial_{t} u+a_{\star} \partial_{x} r=\omega v \\
\partial_{t} v=-\omega u
\end{array}\right.
$$

where $a_{\star}$ and $\omega$ are constants of order $\mathscr{O}(1)$ - respectively related to the wave velocity and to the rotating velocity $-r$ is the first order perturbation of the water depth $h$ and $(u, v)$ is the leading order for the velocity field. The stationary state corresponding to System (2) is the $1 \mathrm{~d}$ version of the so-called geostrophic equilibrium and is given by

$$
u=0, \quad a_{\star} \partial_{x} r=\omega v .
$$

A first study of the accuracy of numerical schemes applied to system (2) for initial data that are close to the kernel (3) was performed in [2]. It was shown that the standard Godunov scheme applied to the linear wave equation with Coriolis source term is inaccurate at low Froude number and the numerical viscosity on the pressure equation is the main reason for this inaccuracy. A modified low Froude Godunov scheme was proposed to cure the problem. The scheme was shown to be $L^{2}$ stable 
under a suitable CFL condition. The proofs extend the ideas introduced in [5] for the study of the homogeneous wave equations in low Mach number regimes.

In this paper, our objective is to study in the same context the numerical scheme introduced in [3] as a well-balanced (WB) scheme for the Shallow Water equations with Coriolis source term (1). In particular we prove the $L^{2}$ stability of the scheme under suitable CFL conditions. Moreover we compare this scheme, called apparent topography scheme in the following, and the low Froude one in terms of dispersion relations and accuracy for some test cases. Note that a high order extension of the apparent topography scheme for the non-linear SW equations with Coriolis source term has been studied in [4], where the authors also paid attention to the linear dispersion relation (hence related to (2)).

\section{The numerical schemes}

Both low Froude and apparent topography schemes are colocated finite volume schemes and can be interpreted as a way to modify the numerical diffusion of the classical Godunov scheme on the pressure equation. In the low Froude scheme proposed in [2], the numerical diffusion on the pressure equation is simply deleted. In the apparent topography scheme introduced in [3], the diffusion term of the classical Godunov scheme remains and an additional consistent term is introduced in the pressure equation such that the numerical diffusion vanishes when applied to an element of the linear kernel (3). The name apparent topography comes from the fact that the scheme was first developed in the context of WB methods for the shallow water equation with topography, see [1]. The two aforementioned semi-discrete schemes applied to (2) read

$$
\left\{\begin{array}{l}
\frac{\mathrm{d}}{\mathrm{d} t} r_{j}+a_{\star} \frac{u_{j+1}-u_{j-1}}{2 \Delta x}-\frac{\kappa_{r} a_{\star} \Delta x}{2} \frac{r_{j+1}-2 r_{j}+r_{j-1}}{\Delta x^{2}}+\frac{\kappa_{r} \omega}{2} \frac{v_{j+1}-v_{j-1}}{2}=0, \\
\frac{\mathrm{d}}{\mathrm{d} t} u_{j}+a_{\star} \frac{r_{j+1}-r_{j-1}}{2 \Delta x}-\frac{\kappa_{u} a_{\star} \Delta x}{2} \frac{u_{j+1}-2 u_{j}+u_{j-1}}{\Delta x^{2}}=\omega f\left(v_{j-1}, v_{j}, v_{j+1}\right), \\
\frac{\mathrm{d}}{\mathrm{d} t} v_{j}=-\omega f\left(u_{j-1}, u_{j}, u_{j+1}\right) .
\end{array}\right.
$$

where the low Froude scheme corresponds to the choice $\kappa_{r}=0, f(x, y, z)=y$ and the apparent topography scheme to the choice $\kappa_{r}=\kappa_{u}, f(x, y, z)=\frac{x+2 y+z}{4}$.

\subsection{Study of the semi-discrete scheme - Dispersion relations}

We now study the stability of the semi-discrete Godunov type schemes by means of Fourier modes:

$$
r_{j}(t)=\varphi_{r}(t) e^{i k x_{j}}, \quad u_{j}(t)=\varphi_{u}(t) e^{i k x_{j}} \quad \text { and } \quad v_{j}(t)=\varphi_{v}(t) e^{i k x_{j}} .
$$


Substituting these expressions into (4), we obtain the following linear system of differential equations

$$
\left(\begin{array}{l}
\varphi_{r}^{\prime}(t) \\
\varphi_{u}^{\prime}(t) \\
\varphi_{v}^{\prime}(t)
\end{array}\right)+\left(\begin{array}{ccc}
\kappa_{r} a_{\star} \frac{\sin ^{2}\left(\frac{k \Delta x}{2}\right)}{\frac{\Delta x}{2}} & i a_{\star} \frac{\sin (k \Delta x)}{\Delta x} & i \frac{\mathcal{K}_{r} \omega \Delta x}{2} \frac{\sin (k \Delta x)}{\Delta x} \\
i a_{\star} \frac{\sin (k \Delta x)}{\Delta x} & \kappa_{u} a_{\star} \frac{\sin ^{2}(k \Delta x / 2)}{\Delta x / 2} & -\omega \zeta \\
0 & \omega \zeta & 0
\end{array}\right)\left(\begin{array}{l}
\varphi_{r}(t) \\
\varphi_{u}(t) \\
\varphi_{v}(t)
\end{array}\right)=\left(\begin{array}{l}
0 \\
0 \\
0
\end{array}\right)
$$

where $\zeta=1$ for the low Froude scheme and $\zeta=\cos ^{2}\left(\frac{k \Delta x}{2}\right)$ for the apparent topography scheme. The first eigenvalue of the amplification matrix is $\lambda=0$, corresponding to the discrete stationary state (3). The other two, corresponding to the inertia-gravity modes, are given in Table 1 . Their real part $\Re(\lambda)$ characterizes the decay of Fourier modes $k$. Since $\Re(\lambda)>0$, both low Froude and apparent topography schemes are damping. The damping rate of the apparent topography scheme is twice larger than that of the low Froude scheme. The imaginary part $\mathfrak{I}(\lambda)$ characterizes the propagation properties of the Fourier modes. Note that for the low Froude scheme, the eigenvalues may be real for $k \Delta x$ close to $\pi$ which means the corresponding modes do not propagate and are only damped. For small $a_{*} k / \omega$, the dispersion relation $\mathfrak{I}(\lambda) / \omega$ of the low Froude scheme is closer to the exact one (for the rotating wave equation (2)) whereas the converse holds for large $a_{*} k / \omega$.

\begin{tabular}{cc}
\hline Wave equation & $\pm i \sqrt{a_{\star}^{2} k^{2}+\omega^{2}}$ \\
\hline Low Froude & $a_{\star} \frac{\kappa_{u}}{2} \frac{\sin ^{2}\left(\frac{k \Delta x}{2}\right)}{\frac{\Delta x}{2}} \pm i \sqrt{a_{\star}^{2}\left(\frac{\sin (k \Delta x / 2)}{\Delta x / 2}\right)^{2}\left[\cos ^{2}\left(\frac{k \Delta x}{2}\right)-\left(\frac{\kappa_{u}}{2}\right)^{2} \sin ^{2}\left(\frac{k \Delta x}{2}\right)\right]+\omega^{2}}$ \\
Apparent Topography & $a_{\star} \kappa_{u} \frac{\sin ^{2}\left(\frac{k \Delta x}{2}\right)}{\frac{\Delta x}{2}} \pm i \sqrt{a_{\star}^{2}\left(\frac{\sin (k \Delta x)}{\Delta x}\right)^{2}+\omega^{2}\left(\frac{1+\cos (k \Delta x)}{2}\right)^{2}}$ \\
\hline
\end{tabular}

Table 1: The eigenvalues corresponding to the inertia-gravity modes for small $k \Delta x$.

\subsection{Study of the fully discrete scheme: kernel and $L^{2}$-stability}

The fully discrete apparent topography scheme applied to (2) can be written as

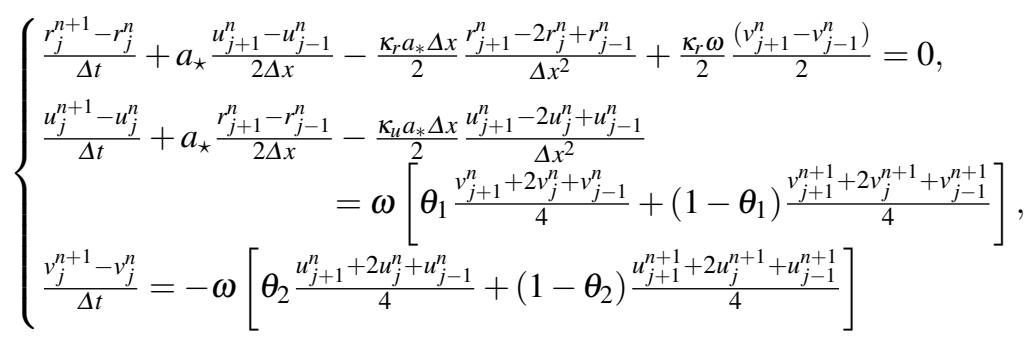




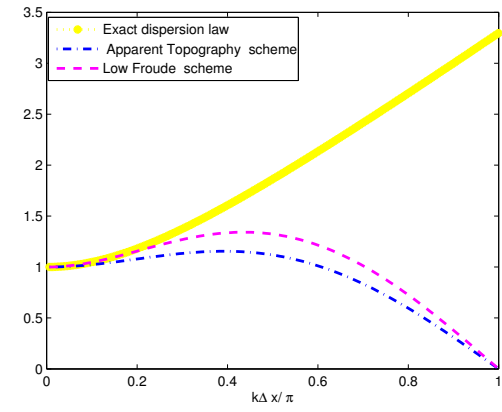

(a) Dispersion relation $\mathfrak{I}(\lambda) / \omega$

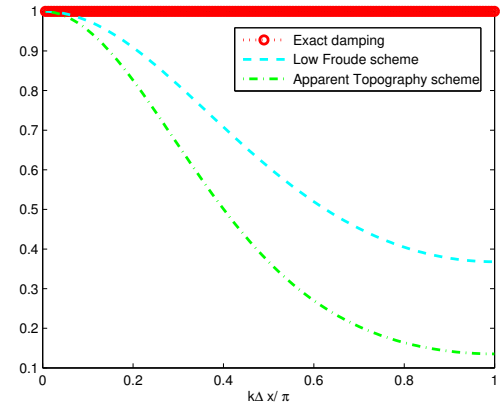

(b) Damping error $e^{-\Re(\lambda)}$

Fig. 1: Numerical properties of the semi-discrete schemes with the Rossby deformation $R_{d}:=\frac{a_{\star}}{\omega}=\Delta x$ and $\left(\kappa_{r}, \kappa_{u}\right)=(0,1)$ for $\mathrm{LF},\left(\kappa_{r}, \kappa_{u}\right)=(1,1)$ for AT.

for $j \in\{1, \ldots, N\}$ and $0 \leq \theta_{1}, \theta_{2} \leq 1$. Setting $q=(r, u, v)$, periodic boundary conditions read $q_{0}^{n+1}=q_{N}^{n+1}, q_{N+1}^{n+1}=q_{1}^{n+1}$. For practical reasons, we assume that the cell number $N$ is odd.

Lemma 1. The kernel of the Apparent Topography scheme (6) is given by

$$
\mathscr{E}_{\omega \neq 0}=\operatorname{ker} L_{\kappa, h}=\left\{q=(r, u, v) \mid u_{j}=0, a_{\star} \frac{r_{j+1}-r_{j}}{\Delta x}=\omega \frac{v_{j+1}+v_{j}}{2}\right\} .
$$

Proof. A stationary state has to satisfy relations (6) with $q^{n+1}=q^{n}$. In particular, the third equation, with an odd number of points and given periodic boundary conditions, leads to

$$
u_{j}^{n}=0, \quad \forall j \in\{0, \ldots, N+1\} .
$$

We then deduce from the first two relations that

$a_{\star} \frac{r_{j+1}^{n}-2 r_{j}^{n}+r_{j-1}^{n}}{\Delta x}=\omega \frac{v_{j+1}^{n}-v_{j-1}^{n}}{2}$ and $a_{\star} \frac{r_{j+1}^{n}-r_{j-1}^{n}}{\Delta x}=\omega \frac{v_{j+1}^{n}+2 v_{j}^{n}+v_{j-1}^{n}}{2}$.

Summing the two equations yields the discrete kernel (7). Conversely, any element satisfying (7) is a stationary state of relations (6). This discrete kernel is a consistent discretization, defined at the cell interfaces, of the continuous kernel (3).

Remark 1. Let us recall that the discrete kernel of the low Froude scheme is

$$
u_{j}=0, \quad a_{\star} \frac{r_{j+1}-r_{j-1}}{2 \Delta x}=\omega v_{j}
$$

(see [2]) which is another consistent discretization, defined at the cell centers, of the continuous kernel (3). 
Remark 2. When the number of points is even, checkerboard modes for velocity $u$ may exist in the discrete kernel of the apparent topography scheme. Note that the low Froude scheme suffers the same drawback, but for the pressure $r$.

We will now investigate the $L^{2}$ stability of the apparent topography scheme. Let us first mention that when $0<\theta_{1}, \theta_{2}<1$, the apparent topography scheme requires to solve a linear system at each time step, which leads to an additional computational cost. On the other hand, the case $\theta_{1}=\theta_{2}=1$, that corresponds to a fully explicit scheme, is known to be unstable - see [4]. Therefore, we restrict our study to the two cases $\theta_{1}=0, \theta_{2}=1$ and $\theta_{1}=1, \theta_{2}=0$. Note that in [2], the $L^{2}$ stability of the low Froude scheme was studied for all values of $\left(\theta_{1}, \theta_{2}\right) \in[0,1]^{2}$.

Lemma 2. Under the hypothesis

$$
\kappa_{r} \kappa_{u} \leq 1+\frac{\omega^{2} \Delta x^{2}}{4 a_{\star}^{2}}
$$

the apparent topography scheme is $L^{2}$ stable under the CFL condition

$$
\Delta t \leq \min \left\{\Delta t_{a}, \Delta t_{b}, \Delta t_{c}\right\}
$$

where

$$
\Delta t_{a}:= \begin{cases}\frac{-\frac{\left|a_{\star}\right|}{\Delta x}+\sqrt{\frac{a_{\star}^{2}}{\Delta x^{2}}+\left(\kappa_{r}+\kappa_{u}\right) \kappa_{r} \omega^{2}}}{\kappa_{r} \omega^{2}} & \text { if } \kappa_{r} \neq 0, \\ \frac{\kappa_{u}}{2} \frac{\Delta x}{\left|a_{\star}\right|} & \text { otherwise. }\end{cases}
$$

and

$$
\Delta t_{b}:=\min \left\{\frac{1}{\kappa_{r}}, \frac{1}{\kappa_{u}}\right\} \frac{\Delta x}{\left|a_{\star}\right|}, \quad \Delta t_{c}:=\frac{2}{\omega}
$$

Remark 3. Note that the choice $\kappa_{r}=0$ is similar to the low Froude scheme, but with a discretization of the Coriolis term at the interfaces. We then retrieve the same CFL condition as that of the cell-centered low Froude scheme, see [2].

Remark 4. Hypothesis (8) is not restrictive since the low Froude scheme always satisfies this condition and the classical choice for the apparent topography scheme is to take $\kappa_{r}=\kappa_{u}=1$.

Remark 5. The bound $\Delta t_{c}$ is the classical CFL condition for the inertial oscillations phenomenon.

Remark 6. The bound $\Delta t_{b}$ is one of the classical CFL conditions for the problem without rotation. For $\Delta x \ll 1$, the asymptotic expansion of the bound $\Delta t_{a}$ leads to the other classical CFL condition for the problem without rotation

$$
\Delta t_{a}=\frac{\kappa_{r}+\kappa_{u}}{2} \frac{\Delta x}{\left|a_{\star}\right|} .
$$


Proof. We perform a Von Neumann analysis to investigate the stability condition. Let us denote

$$
\sigma=\frac{\Delta t}{\Delta x}, \quad \gamma=\omega \Delta t, \quad s=\sin \left(\frac{k \Delta x}{2}\right), \quad \mu=\cos ^{2}\left(\frac{k \Delta x}{2}\right)=1-s^{2} .
$$

By substituting the discrete Fourier modes $r_{j}^{n}=\varphi_{r}^{n} e^{i k x_{j}}, u_{j}^{n}=\varphi_{u}^{n} e^{i k x_{j}}$ and $v_{j}^{n}=$ $\varphi_{v}^{n} e^{i k x_{j}}$ into the fully discrete scheme (6), we obtain $\mathscr{A} \varphi^{n+1}=\mathscr{B} \varphi^{n}$ where the matrices $\mathscr{A}$ and $\mathscr{B}$ are given by

$$
\mathscr{A}=\left(\begin{array}{ccc}
1 & 0 & 0 \\
0 & 1 & -\left(1-\theta_{1}\right) \gamma \mu \\
0 & \left(1-\theta_{2}\right) \gamma \mu & 1
\end{array}\right)
$$

and

$$
\mathscr{B}=\left(\begin{array}{ccc}
1-2 \kappa_{r}\left|a_{\star}\right| \sigma s^{2} & -a_{\star} \sigma i \sin (k \Delta x) & -\frac{\kappa_{r} \omega \Delta t}{2} i \sin (k \Delta x) \\
-a_{\star} \sigma i \sin (k \Delta x) & 1-2 \kappa_{u}\left|a_{\star}\right| \sigma s^{2} & \theta_{1} \gamma \mu \\
0 & -\theta_{2} \gamma \mu & 1
\end{array}\right) .
$$

We then search for the eigenvalues of the amplification matrix $\mathscr{C}=\mathscr{A}^{-1} \mathscr{B}$, that are the roots of the third order polynomial $\mathscr{P}(\lambda)=\operatorname{det}(\mathscr{B}-\lambda \mathscr{A})$. Easy computations lead to

$$
\mathscr{P}(\lambda)=(1-\lambda)\left(\Lambda \lambda^{2}+\xi \lambda+\zeta\right)
$$

with

$$
\begin{aligned}
& \Lambda=1+\gamma^{2} \mu^{2}\left(1-\theta_{1}\right)\left(1-\theta_{2}\right)>0 \\
& \xi=-2+\gamma^{2} \mu^{2}\left(\theta_{1}+\theta_{2}-2 \theta_{1} \theta_{2}\right) \\
& +2\left(\kappa_{r}+\kappa_{u}\right)\left|a_{\star}\right| \sigma s^{2}+2 \kappa_{r}\left|a_{\star}\right| \sigma s^{2} \gamma^{2} \mu^{2}\left(1-\theta_{1}\right)\left(1-\theta_{2}\right) \\
& \zeta=1+\gamma^{2} \mu^{2} \theta_{1} \theta_{2}-2\left(\kappa_{r}+\kappa_{u}\right)\left|a_{\star}\right| \sigma s^{2} \\
& +4 a_{\star}^{2} \sigma^{2} s^{2}\left(1-s^{2}\right)+4 \kappa_{r} \kappa_{u} a_{\star}^{2} \sigma^{2} s^{4}+2 \kappa_{r}\left|a_{\star}\right| \sigma s^{2} \gamma^{2} \mu^{2} \theta_{2}\left(1-\theta_{1}\right) .
\end{aligned}
$$

The eigenvalue $\lambda_{0}=1$ corresponds to the discrete kernel (7). In order to ensure that the other two roots of (9) are in the unit circle $\left(\left|\lambda_{ \pm}\right| \leq 1\right)$, the coefficients $\Lambda, \xi$ and $\zeta$ have to satisfy $|\zeta| \leq \Lambda$ and $|\xi| \leq \Lambda+\zeta$. Computations are then similar to the ones in [2] and lead to the results. More precisely, condition $\zeta \leq \Lambda$ leads to the condition involving $\Delta t_{a}$ and condition $|\xi| \leq \Lambda+\zeta$ leads to conditions involving $\Delta t_{b}$ and $\Delta t_{c}$.

\section{Numerical results}

Let us fix the parameters $a_{\star}=1, \omega=1, \theta_{1}=1, \theta_{2}=0$ and consider the initial condition on the domain $(0,2 \pi)$ 


$$
q_{i}^{0}=\hat{q}_{i}^{0}+M \frac{\tilde{q}_{i}^{0}}{\left\|\tilde{q}_{i}^{0}\right\|} \quad \text { where }\left\{\begin{array}{l}
\hat{q}_{h}^{0}(x)=\left(\sin (\omega x), 0, a_{\star} \cos (\omega x)\right) \in \mathscr{E} h, \\
\tilde{q}_{h}^{0}(x)=\left(a_{\star} \cos (\omega x), 1, \sin (\omega x)\right) \in \mathscr{E}_{\omega \neq \perp},
\end{array}\right.
$$

which is close to the kernel $\mathscr{E}_{\omega \neq 0}^{h}$ up to a perturbation of order $M$. We solve the 1D linear wave equation (2) by means of the Apparent Topography scheme (6), the low Froude scheme and the classical Godunov scheme. We observe on Figure 2 (left) that the classical Godunov scheme is inaccurate since the deviation from the kernel does not remain of order $M$, while the two schemes designed for the geostrophic regime have the correct behavior as the Froude number goes to 0 . We now investigate the accuracy with time at a fixed Froude number. As exhibited for the semidiscrete scheme, we see on Figure 2 (right) that the Apparent Topography scheme is more diffusive than the low Froude scheme for the part of the signal which is in the orthogonal of the kernel.

In future works, the authors will apply the two schemes to linear $2 \mathrm{D}$ cases before considering nonlinear applications in order to discriminate them.

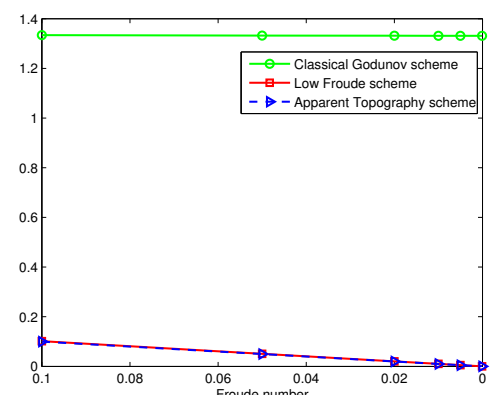

(a) Maximum in time of the deviation $\left\|q_{h}-\hat{q}_{h}^{0}\right\|$ depending on the Froude number

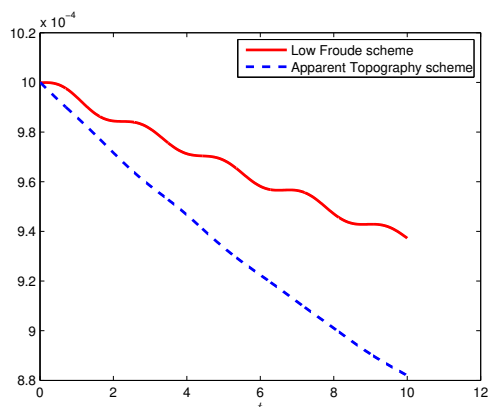

(b) Evolution of the deviation $\left\|q_{h}-\hat{q}_{h}^{0}\right\|$ for $M=10^{-3}$

Fig. 2: Comparisons of classical and WB schemes

\section{References}

1. Audusse, E., Bouchut, F., Bristeau, M.O., Klein, R., Perthame, B.: A fast and stable wellbalanced scheme with hydrostatic reconstruction for shallow water flows. SIAM J. Sci. Comput. 25(6), 2050-2065 (2004)

2. Audusse, E., Dellacherie, S., Do, M.H., Omnes, P., Penel, Y.: Godunov type scheme for the linear wave equation with Coriolis source term. Accepted in ESAIM:ProcS.

3. Bouchut, F., Le Sommer, J., Zeitlin, V.: Frontal geostrophic adjustment and nonlinear wave phenomena in one-dimensional rotating shallow water. II. High-resolution numerical simulations. J. Fluid Mech. 514, 35-63 (2004)

4. Castro, M.J., López, J.A., Parés, C.: Finite volume simulation of the geostrophic adjustment in a rotating shallow-water system. SIAM J. Sci. Comput. 31(1), 444-477 (2008)

5. Dellacherie, S.: Analysis of Godunov type schemes applied to the compressible Euler system at low Mach number. J. Comput. Phys. 229(4), 978-1016 (2010) 